\title{
Acrónimo para una arquitectura orientada por objetos
}

\author{
Acronym for an object oriented architecture
}

Mgt. Mauricio Muñoz Escalante munoz.mauricio@gmail.com

Universidad Antonio Nariño Colombia

\section{Resumen}

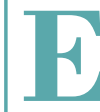

I propósito de este ensayo es promover reflexiones sobre el objeto arquitectónico a partir de la lectura que propone Graham Harman, Manuel DeLanda, Bruno Latour y otros filósofos libremente asociados con una nueva teoría sobre los objetos en la cultura actual. Se muestra la preexistencia de objetos arquitectónicos desde una perspectiva moderna anterior, así como la relación intrínseca entre objeto y sujeto, para luego analizar cómo una ontología plana como la sugerida por la Ontología Orientada por Objetos, cambia los paradigmas del todo formado por la suma de sus partes, así como la correlación entre los objetos y el ser humano, entre otras. Se defiende la idea de que la existencia de los objetos a pesar de las relaciones entre las partes produce un sinfín de imágenes o apariencias del objeto, pero éste en-sí-mismo permanece inaccesible a nosotros. En ese orden de ideas, se concluye con una especulación sobre la imposibilidad de un acrónimo que pretenda contener tanta multiplicidad.

Palabras clave: Ontología Orientada por Objetos, teoría arquitectónica, filosofía en relación a la arquitectura, estética.

\section{Abstract:}

The main purpose of this essay is to promote a reflection on architectural objects apropos Graham Harman, Manuel DeLanda, Bruno Latour and other philosophers' loosely associated theory about objects on actual culture. It presents how architecture objects were already present from a modern previous perspective, as well as its intrinsic object-subject relation, and then it analyzes how a flat ontology, as proposed by Object Oriented Ontology, changes paradigms such as the whole as sum of its parts, and the correlation between human beings and objects, among others. It supports the idea that the existence of objects despite its relations produces an endless amount of images or appearances, while the object-in-itself remains inaccessible to us. Accordingly, it finally speculates that an acronym containing such multiplicity would also be impossible.

Keywords: Object Oriented Ontology, architecture theory, philosophy in relation to architecture, aesthetics 


\section{Introducción}

Aunque son innegables los esfuerzos de los críticos latinoamericanos por orientar el discurso arquitectónico hacia problemáticas regionales, es así mismo innegable la fuerza con la que las teorías anglosajonas azotan en especial las escuelas de arquitectura del continente, donde los jóvenescon frecuencia más expuestos a los medios masivos, en su mayoría también anglosajonespromueven a través de sus proyectos de diseño lenguajes cada vez más sofisticados formal y técnicamente hablando, sin que en muchas ocasiones se les pueda dar el debate necesario, asunto que deja por el camino no solo baches en la consolidación de un cuerpo conceptual más homogéneo, sino edificios aislados que no pueden insertarse con facilidad en el tejido urbano (fallan en la práctica), y tampoco encuentran una argumentación satisfactoria (fallan en la teoría).

En ese sentido, la reflexión que se plantea a continuación tiene como propósito salirle al paso a la Ontología Orientada por Objetos, una corriente filosófica aún en ciernes en Estados Unidos y Europa, que ya atrae la producción de nuevas generaciones de arquitectos, y ya suscita mucha discusión en las facultades de arquitectura más vanguardistas del mundo, tal como la Architectural Association de Londres (Latour, 2011), el Pratt Institute de Nueva York (Schumacher, 2012), el Instituto de Arquitectura del Sur de California (Ruy, 2013) y la Escuela de Arquitectura de Yale (Gage, 2014).

De esa manera se busca evitar en lo posible que los referentes arquitectónicos lleguen de nuevo a América Latina empaquetados con la teoría y no haya tiempo de separar, como se dice coloquialmente, el bebé del agua sucia. Basta recordar dos ejemplos anteriores: la filosofía de Jaques Derrida, que se presentó ya con las Ciudades de arqueología ficticia de Peter Eisenman a finales de los años ochenta del siglo XX; y la filosofía de Gilles Deleuze, que apareció una década después con el terminal internacional de pasajeros de Yokohama diseñado por Foreign Office Architects. En ambos casos la crítica latinoamericana se vio abocada a improvisar por el camino sobre una propuesta plástica final-o por lo menos muy avanzada-y solo pudo resistir la entonces nueva materialización espacial de la filosofía, como ocurrió con la arquitectura deconstructivista (Waisman, 1989); o se tuvo que contentar con repetir los ejercicios proyectuales originales varios años después, como los que se hicieron en Argentina, Chile y Brasil alrededor del pliegue (Chiarella, 2011).

En el caso de la Ontología Orientada por Objetos no hay arquitectura ligada aún a ella, o por lo menos no se evidencia tan claramente. Puede estar en las propuestas de Mark Foster Gage o en algunas obras de Ali Rahim o François Roche, pero es palmario que estamos ante una transición en la que todavía se pueden encontrar puntos de contacto para enriquecer el discurso y no necesariamente permanecer al margen, como en el pasado.

Las razones que se exploran al respecto son cuatro: 1) que filosóficamente la Ontología Orientada por Objetos se enmarca en la búsqueda de un nuevo materialismo a partir de un distanciamiento del concepto de correlación de Kant, en el cual el conocimiento solo emerge mediado por el pensamiento (Meillasoux, 2008), lo que beneficia la tesis de un eclecticismo regional que hasta ahora no se define sino a grandes rasgos (Gutiérrez y Gutiérrez-Viñuales, 2012); 2) que socialmente se inserta en el aparato teórico posmoderno, que promueve la emergencia de discursos alternativos originarios de poblaciones que han permanecido en la periferia, como la latinoamericana; 3) que académicamente no entra de la mano de autores célebres como Derrida y Deleuze, sino que se instala en el imaginario con la tecnología, que al estar al alcance de cualquiera se oculta perfectamente a plena luz del día, como lo hace la Internet de las Cosas, que según sus mercaderes ya es una realidad incluso en América Latina; y 4) que culturalmente la Ontología Orientada por Objetos se presenta al mundo contemporáneo como un acrónimo-OOOasunto que le provee un espíritu global que puede encontrar resonancias en un pensamiento que se aleja aposta de los cánones metafísicos y epistemológicos occidentales tradicionales (Candia, 2007).

\section{Cuando los objetos tenían su propio reino}

La Internet de las cosas (Internet of Things-10T) se define comúnmente como "la interconexión de objetos equipados con inteligencia ubicua". (Xia, Yang, Wang y Vinel, 2012, p. 1101).

Esta acepción tan sencilla de la loT (llamada también Internet de los objetos) confirma el dogma actual que ignora todo lo que no sea identificable por la tecnología bajo la premisa de que todo es información (Vedral, 2010). Así se manifiesta en la publicidad de una reconocida empresa latinoamericana en la que hombres sonrientes vigilan desde su teléfono celular a sus esposas, hijos, mascotas y propiedades materiales, ya sean éstas carros, bicicletas o morrales (Claro, 2017a y 2017b). La única que 
aparentemente falta es la arquitectura. En alguno de esos volantes se ven unos artículos encaramados en estibas, pero se intuye que la preocupación no es la bodega el objeto arquitectónico, pues nadie se lo puede llevar a ninguna parte- y que más bien lo que se protege es la mercancía, a lo mejor valiosa y cubierta por algún seguro contra robo o terrorismo. Pero entonces, ¿qué pasa con los edificios?

Por ahora se pueden inferir tres argumentos:

1) Que los humanos, los animales y los artículos cotidianos son todos objetos para la loT;

2) Que los objetos pertenecientes a la loT no tienen jerarquía, sino que seres humanos, animales y artículos cotidianos están todos al mismo nivel; y

3) Que todo lo que no disponga de dicha inteligencia ubicua no es parte de la loT.

Desde una perspectiva moderna, la propuesta es turbadora pues los objetos existían justamente para distinguir lo creado por la naturaleza de los artículos producidos por el hombre: a un lado, animales, plantas, rocas; y al otro, edificios, vestidos, juguetes. De ahí la dicotomía natural/artificial. Comprender esa primera suposición de la loT (que los seres que entendíamos como vivientes también son objetos) solo es concebible en el marco de un desarrollo tecnológico que diluya dicha división entre lo natural y lo artificial. Piénsese en las series de algoritmos, las secuencias de ADN, los códigos computacionales, y los lenguajes de redes que de una u otra manera fueron revelando que las diferencias entre humanos y cerdos, y entre cerdos y granos de arroz no son tan profundas como pensábamos y que por el contrario compartimos una gigantesca infraestructura común. Basta recordar las incesantes cadenas verdes de unos y ceros que en 1999 definieron por igual al cursor titilante del monitor y al cable de fibra óptica y a los muros del edificio y a Neo y a Morpheus y a Trinity, todos formando parte de un gran organismo universal Ilamado Matrix (Thill, 2011)

Siguiendo esa misma línea es posible coincidir con la IoT, en una segunda instancia, en que el ser humano "es simplemente una clase de entidad entre trillones de otras" (Harman, 2010a, p. 772), entre las cuales se incluyen, por supuesto, los objetos arquitectónicos.

El problema aparece en la tercera suposición: no porque se deba probar que la arquitectura tenga inteligencia ubicua, pues habría que hacer lo mismo con todo lo demás: vestidos, juguetes, etc. A la luz de la misma perspectiva moderna, dicha inteligencia ubicua no hacía parte de los objetos, claro que no, pero con la actual tecnología disponible sí que se puede vaticinar, como hace el futurista Ray Kurzweil (Ptolemy y Ptolemy, 2009), que todo será inteligente. Se dice que representa un problema porque si la inteligencia ubicua se le impone al objeto, es virtualmente imposible pensar que exista la posibilidad de ser o no parte de la loT. Naturalmente, para que la loT funcione, todo debe hacer parte de ella (Marr, 2018). Nada debe quedar por fuera. En ese sentido, no es que la loT aparezca en el mercado de la mano de las intenciones mercantiles de las compañías de telecomunicaciones y las películas de ciencia ficción, y para funcionar convierta todo en objetos, sino que todo ya era un objeto y la loT solo aprovecha esa condición para funcionar. De esa manera, aunque podría afirmarse que todo lo que no es parte de la loT no es un objeto - éste podría volver a su estado anterior de vestido o juguete-, ése parece un postulado retórico, alojado tal vez en la utopía.

\section{Lo que era el objeto arquitectónico}

La primera reacción es decir que la arquitectura ya era un objeto: si algo pertenecía a la loT, desde antes incluso de que existiera la loT, eran los objetos arquitectónicos:

Como estrategia retórica en la que "objeto" se intercambia indistintamente con otras palabras que designan productos arquitectónicos, como por ejemplo, "obra":

Si bien el crítico debe analizar y contextualizar el edificio dentro de un marco teórico que permita entender su razón de ser, detrás de la obra arquitectónica en sí misma debe subyacer una teoría que justifique por qué el objeto final es ése y no otro. (Muñoz, 2007, p. 55);

Como nombre específico para referirse a los productos creativos de los arquitectos:

Puede ser que la expresión "objeto arquitectónico» este cargada de demasiado significado. Sin embargo, esto es menos probable que con otras alternativas comunes que ofrece la literatura: "obra de arquitectura» está muy comprometido con la intención del creador, su estatus como un todo íntegro o vigente, y su rango estético; «Edificio» ciertamente está ligado a lo concreto, a los inmuebles erigidos; y «Estructura» se usa para designar resultados construidos genéricos que resultan del diseño arquitectónico, en reconocimiento a un dominio que algunos ven como más vasto que los edificios per se. (Fisher, 2015, ๆ 2);

Como término universal para designar cualquier construcción real:

Los medios de representación y de comunicación entonces se convierten precisamente en medios de producción, con la esperanza de que en este cambio el dibujo siga siendo e espejo perfecto que permita el paso ininterrumpido de idea a objeto arquitectónico. (Till, 2009, p. 111);

Como el todo producto de sus partes:

Los arquitectos nos ocupamos de diseñar moradas, en tanto que manifestaciones arquitectónicas de espacio, estructura y orden, pero no parecemos capaces de tocar los aspectos más sutiles, emocionales y difusos del hogar. [Esto se debe a que] nuestro concepto de arquitectura está basado en la idea de un objeto arquitectónico perfectamente articulado. (Pallasmaa, 1992, p. 1);

Como el otro en el dualismo entre espectáculo-espectador/adentro-afuera:

Las fantasías imaginadas por el urbanismo modernista europeo (por ejemplo, la fantasía urbanoarquitectónica de Le Corbusier de una ciudad de torres de vidrio en un parque, con calles anchas sobre un patrón reticular, donde 
la gente camina sobre andenes elevados) muestran la imposible relación de la arquitectura con su objeto-causa-de-deseo: la ciudad. (Gandelsonas, 1998, p. 129);

Y en el sentido ontológico: la casa, el colegio, el parque, la oficina - los espacios que albergan las personas, animales y cosas - tienen una materialidad y en tanto, son, existen, son objetos:

Si la ontología es la teoría de los objetos y sus relaciones - una estructura dentro de la cual a ser en sí mismo se le puede dar alguna organización- entonces creo que e arte, en general, y la arquitectura, en particular, pueden y operan ontológicamente. (Hays, 2004, p. 2).

Incluso en el sentido específico del comercial citado arriba, en el que la loT funciona como una red que permite localizar e incluso operar todas las cosas a distancia, los edificios ya eran también objetos de vigilancia por medio de circuitos cerrados de televisión y sistemas de monitoreo satelital: lo innovador ahora es que la loT incluya todo lo demás - la nevera y la cortina y las luces inteligentes sumidas dentro del cielorrasosiguiendo los sueños futurísticos de programas como Los supersónicos, donde Súper atendía a su jefe desde su casa, Ultra preparaba el desayuno de su Cometín sin levantarse de la silla, y Robotina era parte de la familia como cualquier otro ser.

\section{Lo que era la relación entre objeto y sujeto}

Para la arquitectura, la dificultad de concebir todo como un objeto surge en la relación hombre-edificio, pues desde los tiempos de Roma ésta fue fundamental para entender y ejercer la disciplina:

Las partes de los templos deben guardar una proporción de simetría perfectamente apropiada de cada una de ellas respecto al conjunto total en su completa dimensión [...] si la naturaleza ha formado el cuerpo humano de modo que sus miembros guardan una exacta proporción respecto a todo el cuerpo, los antiguos fijaron también esta relación en la realización completa de sus obras, donde cada una de sus partes guarda una exacta y puntual proporción respecto a la forma total de su obra. (Vitruvio, 1995, p. 82).

La arquitectura imitaba en sus proporciones a hombre por considerarlo perfecto, y por extensión la arquitectura se relacionaba directamente con la naturaleza, considerada la autora de esa perfección. Eso revela una concepción antropocéntrica de la realidad. El mundo no se concebía sino a través del hombre, de lo que su mente y sus sentidos hacían o percibían de éste. En términos de Jacobs y Gabriel (2013)," los objetos eran secundarios a las acciones humanas y solo se les proveía vida y significado por medio de encuentros humanos" ( $p$ 213). Estos" encuentros humanos" se hacen evidentes en todos los procesos de la arquitectura:

Durante la concepción (el objeto es el edificio, y el sujeto -el que diseña- es el hombre);

En la experiencia del espacio (el objeto es el edificio y el sujeto - el que tiene sentimientos - es el hombre);
En la disposición de los elementos del espacio (los objetos son esos elementos del espacio - las ventanas, la cubiertas, los muebles - y el sujeto - el que se beneficia de la luz que entra por las ventanas, de la sombra que provee la cubierta, de la comodidad de las sillas y de la cocción de los alimentos en la estufa- es el hombre);

En los cálculos ingenieriles (el objeto es el edificio y el sujeto - el que sabe cuánta firmeza deben tener los elementos estructurales, el que entiende las fuerzas que operan para mantener en pie la construcción - es el hombre);

En los valores estéticos que se le otorgan al espacio (e objeto es el edificio y el sujeto -el que considera qué es bello y qué es feo, qué tiene orden y qué no- es el hombre);

En la función en relación a la forma (el objeto es el edificio y el sujeto - el que se inventa, por ejemplo, cómo son las circulaciones en un hospital y las salidas de emergencia en un auditorio - es el hombre);

En el significado del espacio (el objeto es el edificio y el sujeto -el que otorga el rol social, el que le brinda relevancia política o cultural- es el hombre);

Etcétera.

Pensar que el hombre es otro objeto y no el sujeto, es inquietante. Imaginar que el hombre no es el que diseña, vive, dispone, determina, concede, impone y define, sino que todo - los espacios (como el hospital y el auditorio) los elementos estructurales (como las vigas y columnas); los artefactos (como las sillas y la estufa); las fuerzas que operan para mantener en pie la construcción (como la tensión y la compresión); los conceptos compositivos (como el orden y el equilibrio); los fenómenos físicos (como la luz que entra por las ventanas y la sombra que provee la cubierta); los sentimientos (como la comodidad de las sillas y la satisfacción por la ingesta de los alimentos cocidos); y las ideas (como el rol social o la relevancia política y cultural) - si todos son objetos por igual, esto requiere un cambio radical en nuestra manera de entender el mundo.

La pregunta es por qué la humanidad habría de considerarse a sí misma como cualquier otro objeto después de pasar miles de años intentando crear una categoría solo para ella, lejos de las cosas, los animales y las plantas para dominar el mundo, y la respuesta es, "Por eso mismo". Así lo explica Slavoj Zizek:

[Porque] en el preciso instante en que llegamos a se lo suficientemente poderosos como para afectar las condiciones más básicas de nuestra vida, tenemos que aceptar que somos tan solo otra especie animal en un pequeño planeta. Una vez nos demos cuenta de esto, se necesita una nueva forma de relacionarnos con nuestros alrededores: no seguir siendo el trabajador heroico que expresa su potencial creativo usurpando los inextinguible recursos del ambiente, sino un agente mucho más modesto colaborando con su contexto, negociando permanentemente niveles tolerables de seguridad y estabilidad (Zizek, 2017). 
La razón es la consciencia ecológica, la que nos permite conectar los puntos y darnos cuenta de que todo está interconectado (Morton, 2010). Solo así la actividad humana se entiende como un" agente mucho más modesto" para lograr relaciones simbióticas. Sin embargo, para entender cómo este agente" colabora con su contexto", tal vez se necesite concebir un modelo de realidad en la que coexistan objetos de todo tipo: hombres, animales, plantas, minerales, edificios, máquinas y seres no biológicos.

\section{Cuando los objetos se rebelaron}

Si ontología se define como el estudio del ser en tanto que es, como en la máxima de Descartes "Pienso, luego existo", se entiende que incluso ésta frase está inscrita en un modelo antropocéntrico al haber sido pensada por un ser humano. Pero si todo es en tanto existe, como asume la loT, no importa si dichas entidades que existen piensan o no, son artificiales o naturales, o son simbólicas o físicas. En este caso se trata de una ontología fuera del modelo antropocéntrico, o sea, una "ontología plana" (Bryant, 2011, p. 245)

Aunque se asume que el debate filosófico en este sentido se remonta a Aristóteles (Matthews, 1990), la perspectiva actual encuentra su referente principal en Alfred Whitehead, cuando en los años veinte del siglo pasado revolucionó la correspondencia entre el hombre y el mundo al sugerir que la relación entre la prisión y los internos no era más importante que la relación entre los ladrillos de las paredes de la misma prisión o la relación entre las ratas de la prisión y los rayos cósmicos que aniquilaban los protones de sus cerebros (Whitehead, 1978, pp. 355-359). La prisión, los ladrillos, las ratas, los rayos cósmicos y sus cerebros debían estar todos en el mismo plano.

Sin embargo, aunque la idea cayó bien entre la comunidad académica de entonces, el planteamiento de Whitehead no pudo aplicarse fácilmente a la realidad, pues reducía demasiado los objetos a sus relaciones $y$, por ende, no daba pie para explicar el cambio, entendido en parte como el surgimiento de nuevas relaciones (Harman, 2009, p. 131). Los ladrillos seguían siendo de la prisión y los cerebros atacados por rayos cósmicos seguían siendo de las ratas: la relación de interdependencia seguía existiendo. En ese sentido, si el ObjetoPrisión necesitaba de los ladrillos para ser, si se añadía un nuevo volumen a la prisión para aumentar la capacidad del penal, por ejemplo, dicho ObjetoPrisión no podía ser el mismo objeto de antes, siendo que ahora tenía más ladrillos y más presos.
La solución que plantea Harman (2002, pp. 228-232) es complementar la visión de Whitehead de poner todos los objetos al mismo nivel con la idea de Heidegger de aislar los objetos de sus relaciones y de cualquier tipo de presencia en general. Así nace entonces la Filosofía Orientada por Objetos (Harman, 2010b), la cual sostiene que el mundo está lleno de una extensa variedad de objetos que entran en contacto unos con otros de manera indirecta, al tiempo que se retiran mutuamente hacia su propia privacidad. Solo así el ObjetoPrisión de Whitehead es el mismo con más o con menos ladrillos, con más o menos presos, y con más o menos ratas. Acordemente, lo que prospera después como Ontología Orientada por Objetos, OOO, considera que todos los objetos existen a pesar de sus relaciones (Bogost, Bryant, Harman y Shaviro, 2010).

Podría argüirse que esa nivelación de todas las entidades es lo que arrastra a la arquitectura hacia la 000 , pues el objeto arquitectónico ya se había diluido entre las dinámicas urbanas y regionales por cuenta de la crisis ecológica para volverse un objeto más de los ecosistemas; otro ser, considerando la vida del edificio y su degradación en el tiempo: un objeto con una función que cumplir, pero no una función como hospital o prisión, como se entendió con el Movimiento Moderno, sino un objeto con una misión en cuanto a consumo de agua y de energía; como elemento del paisaje donde habitan hombres y ardillas y abejas y granos de polen y moléculas de oxígeno y partículas suspendidas de dióxido de carbono, y no como forma material de puntos, líneas, planos y volúmenes, como dijo Kandinsky en 1926. Solo un cambio de mentalidad de esa proporción puede explicar, por ejemplo, que los edificios con plantas de antaño se convirtieran de repente en "bosques verticales", como se le llamó recientemente a la obra de Stefano Boeri en Milán (Holland, 2018).

\section{Cómo volver a ser un objeto arquitectónico}

Tal vez entonces la manera más expedita de empezar un análisis de la arquitectura a la luz de la 000 sea reemplazando una vez más el nombre, cambiando Ontología Orientada por Objetos, por Arquitectura Orientada por Objetos. La paradoja es que dicha estrategia conduce al lenguaje de los computadores:

Arquitectura Orientada a Objetos es un paradigma de diseño basado en la división de responsabilidades para una aplicación o sistema en objetos individuales, reusables y autosuficientes, que cargan consigo su propia información y comportamiento. Un diseño orientado por objetos entiende un sistema como una serie de objetos cooperantes, en lugar de un juego de rutinas o instrucciones de procedimiento (Microsoft, 2009, ๆ 118).

No es necesario anotar que la definición de los desarrolladores de tecnología se asemeja extrañamente a la tesis de la loT y a los planteamientos de la 000 . Esto prueba que el debate hubiera sido imposible en un mundo pre-digital, como sugiere Alexander Galloway 
(2013), asumiendo como inevitable" la coincidencia entre la estructura de los sistemas ontológicos y la estructura de las tecnologías más altamente evolucionadas del capitalismo" (p. 347). Pero siendo así, ¿cómo explicar la obstinada imagen unitaria de los edificios? Porque no necesariamente la primera impresión que produce una prisión es la de millones de ladrillos y ratas y rayos cósmicos y cualquier infinidad de objetos que interactúan entre sí, sino la de un volumen, simple y llanamente. ¿Cómo se produce el todo?

Una ontología de entidades individuales demanda sabe en cada caso cuáles procesos históricos específicos ocasionan un todo [para] explicar la emergencia de instituciones describiendo las interacciones entre los individuos responsables, la emergencia de centros urbanos en términos de las interacciones entre instituciones, y la emergencia de estados nación como la interacción entre ciudades. La idea es ver las instituciones y los centros urbanos no como totalidades abstractas sino como individuos sociales concretos, con el mismo estatus ontológico de seres humanos individuales, con la diferencia de que operan en escalas espaciotemporales más grandes. (DeLanda, 2002, p. 5).

Hay que señalar que no se habla de edificios, sino de "instituciones". En la prisión del ejemplo los "individuos responsables" serían no solo los ya mencionados ladrillos y ratas, sino también los guardianes y los internos y e personal administrativo y los trabajadores de los servicios generales, así como una cantidad de objetos incorpóreos como la oferta laboral y las actividades culturales y deportivas que se ofrecen, entre muchas más, siendo cada una de ellas "entidades individuales". Pero como las relaciones no definen a los objetos - los ladrillos son ladrillos independientemente de la prisión, las ratas son ratas sin importar los ladrillos, e incluso las actividades culturales son actividades culturales aparte de la prisión y de los ladrillos y de las ratas - las relaciones no existen a pesar de los objetos. Lo que "ocasiona el todo" son precisamente los "procesos históricos", o sea, ésas "interacciones entre los individuos responsables". De esa manera el objeto arquitectónico no aparece como edificio, pero tampoco como espacio, contradiciendo tanto las concepciones materialistas como fenomenológicas a las que estábamos acostumbrados en el siglo XX: aparece como imagen.

Cuando una persona dice, por ejemplo, "Esa prisión me da miedo", ella se refiere al objeto como espacio. Pero si la persona dice "Vamos a la prisión", ésta es también un objeto, solo que material. La diferencia es que este último ObjetoMaterialPrisión -las oficinas, las celdas, el gimnasio: todo lo que se considera vulgarmente como arquitectura - tiene una forma, mientras que el ObjetoEspacialPrisión anterior funciona como una entidad incorpórea. Desde el punto de vista de la 000, ambas prisiones gozan "del mismo estatus ontológico de seres humanos individuales". Y puede haber más, miles más, millones más, a partir de las incontables relaciones entre todos los objetos posibles "que ocasionan el todo". Pero no son "totalidades abstractas": ni el ObjetoMaterialPrisión ni el ObjetoEspacialPrisión ni el ObjetoSocialPrisión ni ningún otro ObjetoPrisión son "el todo"; la prisión como totalidad sigue estando fuera de nuestro alcance.

\section{Cuando el todo es menos que las partes}

Por eso los seguidores de la $\mathrm{OOO}$ claman, invirtiendo la máxima Aristotélica según la cual el todo es más que la suma de sus partes, que el todo "es menos" que la suma de sus partes (Timothy Morton, como se citó en Korody, 2016). Donde antes había una prisión, ahora hay por lo menos dos. El todo - la prisión- es menos que la suma de las relaciones entre los guardianes y los internos y el personal administrativo y los trabajadores de los servicios generales y la oferta laboral y las actividades culturales y deportivas que se ofrecen.

Pero si existen millones de prisiones, cada minuto, cada segundo, ¿dónde están? Porque no puede haber millones de prisiones cuantitativamente hablando; cada copia no existe físicamente como registro factual. Sin embargo, cualitativamente sí, como cuadros en una película sobre la prisión, por ejemplo. Cuando vemos o recorremos la prisión solo tenemos acceso a lo que es ella en cierto instante. solo percibimos una imagen, no el todo. El objeto en sí permanece oculto. Bruno Latour (2011) es tajante al respecto: "los edificios en sí mismos no son sustancia corpórea porque nunca son visibles al mismo tiempo al que se están construyendo" (25'15"-27'07"). Para explicarlo cita a Mario Carpo (2001), quien dice que incluso desde cuando la perspectiva fue inventada y empezó a usarse masivamente, se hizo evidente que cada edificio está localizado individualmente y no hay nada que los conecte unos a otros, sino que se comportan como objetos independientes; y después acude a Albena Yaneba (2009) para exaltar la diferencia entre diseñar un edificio y hacerlo, construirlo, pues el resultado no coincide nunca con lo planeado. Dice Latour que una vez el edificio emerge del papel o, incluso, una vez sale de la mente del arquitecto, éste entra en un estado de "duración", de "continuidad"; se instaura en cierta temporalidad que hace que el objeto nunca sea estático sino un "proyecto", que es incidentalmente la manera en la que los proyectistas nos referimos a los edificios. En ese sentido, siguiendo las meditaciones de Bergson sobre la imagen en movimiento, concluye Latour que el objeto arquitectónico no es material sino que es un cuadro en la secuencia que constituye un proyecto; el objeto es un momento de un proceso (lo que explica en parte el carácter efímero que tradicionalmente se le da a la imagen de los edificios).

Lo importante ahora es preguntarse cómo el objeto arquitectónico aparece ante nosotros. Porque en el sentido puramente disciplinar, no tener acceso al edificio como un todo implica su concepción como un devenir entre las relaciones de las partes (la "emergencia de instituciones describiendo las interacciones entre los individuos responsables" que mencionaba DeLanda), pues aunque esa imagen de la prisión está ligada a lo que ésta es - su apariencia-, no tenemos acceso a lo que la prisión es en sí misma. 


\section{Lo que origina el objeto arquitectónico}

Una respuesta tentativa al interrogante es: cuando el objeto falla (Hale, 2013, q 2). Por ejemplo, si hay asbesto en la prisión, éste está matando a los reclusos y guardianes a cada segundo, pero no lo sabemos a cada instante. Eso solo es perceptible si pasamos en cámara rápida los años que vivieron los afectados en la prisión para apreciar el daño. Cuando se observa un solo cuadro (e.g. el día que sirvieron pollo al almuerzo), no se tiene acceso a la relación entre el asbesto de las tejas y los pulmones de los hombres. Esto se debe, según la OOO, a que las partes no se relacionan entre sí con todas sus cualidades al mismo tiempo, sino solo con unas partes de sus partes. Harman (2007) lo explica con la parábola islámica del fuego que quema el algodón, en la que no estarían involucrados todo el fuego y todo el algodón, sino solo aquellas partes necesarias (e.g. la combustibilidad, que es fundamental para la consumación), mientras que otras permanecen alejadas (e.g. el color o el olor, que no inciden en nada). Solo notamos las primeras y no nos damos cuenta de las últimas.

La prisión entonces no aparece ante nosotros, pues a lo que tenemos acceso es solo a una imagen. La prisión reaparece: el sistema carcelario reconoce que existe la prisión cuando se reporta la muerte de internos por exposición a asbesto; la persona sentada frente al televisor identifica la prisión en el noticiero; el director del penal recuerda que existe algo llamado prisión a diferencia, por ejemplo, de oficina y estacionamiento y comedor; los reclusos caen más en cuenta de la prisión como artefacto que los retiene (solicitan traslado y cada día que pasa el edificio se les convierte en un suplicio); y así sucesivamente. Solo se hacen visibles ciertos cuadros (momentos, apariencias, continuidades). El ObjetoPrisión está alejado pues existe aparte "para ser contemplado en la distancia", como escribió Heidegger (1962, p. 98), hablando de un martillo. Así lo explica Graham Harman, a propósito de dicho análisis de equipos y materiales que hace el filósofo alemán:

Cuando uso un martillo, por ejemplo, estoy concentrado en la construcción del proyecto que me propuse y probablemente doy el martillo por sentado. Solo si el martillo es muy pesado o se me resbala de las manos, o si se rompe, tiendo a no darme cuenta de éste. Pero el hecho de que el martillo se puede romper prueba que tiene otras dimensiones aparte de las que yo entiendo [...] Mirar fijamente un martillo no lo suprime en toda su complejidad, pero tampoco lo hace blandirlo en un sitio de construcción o en un campo de batalla. Tanto la teoría como la práctica deforman el martillo en su realidad no aparente. La OOO lleva el argumento un poco más allá para decir que los objetos se afectan entre sí incluso en las interacciones más casuales. Las gotas de lluvia - las ráfagas de viento que corroen el martillo pueden no ser «conscientes» de ello en términos humanos, sin embargo dichas entidades no extinguen la realidad del martillo en menor grado que la práctica o teoría perpetrada por humanos. (Harman, 2012, p. 187).

Igual con los objetos arquitectónicos: se puede decir que el hombre no planea y construye la totalidad del proyecto (digamos que una prisión para terminar con el mismo ejemplo), sino que éste surge a partir de la interacción de ciertos materiales, unas herramientas, unas condiciones ambientales, y unas cualidades humanas, entre muchas otras. No se puede decir que el hombre en su totalidad está planeando y construyendo la prisión, sino que solo lo hacen algunas de sus cualidades, como la fuerza de sus brazos o la habilidad de sus manos; su capacidad para detectar estrellas fugaces en el firmamento o su destreza para tocar el violín, no estarían involucradas. La prisión se va deviniendo por la interacción de ladrillos y ratas y rayos cósmicos, y cemento y hierro y martillos y palas y palustres y lluvia y viento y rayos solares y sudor y lágrimas y sangre (a veces), hasta los muebles y aparatos $\sin$ fin relacionados, pasando por los elementos macro de las ciudades y las regiones metropolitanas y la estructura ecológica principal, terminando en la escala micro y nano de los materiales de su sustancia, más los planos y presupuestos y licencias y mensajes electrónicos e imágenes digitales y programas de diseño asistido por computador, EEP, DWG, XLS, DOC, HTML, JPG, CAD, toda la loT.

\section{Conclusión}

A la luz de la 000 no podría hablarse de Arquitectura Orientada por Objetos, AOO, porque la arquitectura no solo es orientada por objetos (otros objetos la definen), sino también orientada desde objetos (ella define otros objetos). Podría incluso decirse que es Arquitectura Orientada —seguida por cualquier preposición: a, ante, bajo, cabe, con, contra, de, desde, en, entre, hacia, hasta, para, por, según, sin, so, sobre, tras - Objetos. Pero como es absurdo un acrónimo de esa longitud (AOAABCCCDDEEHHPPSSSSTO), más vale decir Arquitectura Orientada $x$ Objetos, AOxO, donde x no es por, en el sentido de "su función de agente de las oraciones en pasiva", como reza el Diccionario de la Real Academia Española, DRAE (2015); ni por, como signo matemático indicativo de multiplicación; sino x (equis), como variante para reemplazarse según sea el caso.

Así le ocurrió al acrónimo de la comunidad de Lesbianas, Gays, Bisexuales, Transexuales e Intersexuales (LGBTI), que terminó siendo insuficiente para abarcar las 68 variaciones de sexo y de género que se reconocieron posteriormente (Gender Agenda, 2014). Por eso se optó por usar el signo más (LGBTI+) para no caer ni en el vulgar asterisco (LGBTI*) ni en el indefinido etcétera (LGBTI...) ni mucho menos en el impronunciable acrónimo (LGBTIQQ2AAAAAAABCCCCDEFGGGGGGGHHHHHHHHHHHIKLMMMMNNNOOOPPPPPPSSSSTTTTTTUUU), que bien hubiera podido ser de 7.658 millones de caracteres $-y$ contando- si acogemos lo que dicen los expertos de que" existen tantas sexualidades como personas en el mundo" (Barrera, 2018, ๆ 6).

Lo interesante, como observa Slavoj Zizek (2018), es que este signo más no solo es la manera expedita de decir "el resto", dejando por fuera incluso a los LGBTI, sino que es una categoría en sí misma:" así como una persona pue- 
de ser trisexual, asexual, bi-género o lo que sea, también

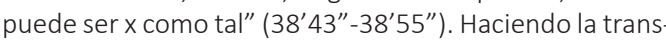
literación, la arquitectura podría ser entonces también directamente solo $\mathrm{x}$.

\section{Recomendaciones}

Esta relectura del objeto arquitectónico no es definitiva, ni mucho menos, pues pensar la arquitectura desde la perspectiva de la 000 es todavía un ejercicio muy especulativo. Baste recordar las palabras de su principal vocero en The Architecture Exchange, un centro de debate entre la arquitectura y la filosofía con base en Gran Bretaña:

No creo que el rol de la filosofía sea qué decir a otras disciplinas. A lo mejor solo funcione como facilitadora de la conversación. No puedo decirles cuál es la manera apropiada de adaptar modelos de 000 a la arquitectura. Se supone que eso lo debo aprender de ustedes [los arquitectos]. Lo que sí puedo decirles es, «Pienso que me han malinterpretado aquí» o «Eso no fue lo que quise decir con eso», pero no tengo ni idea - bueno, tengo alguna idea, pues tengo amigos arquitectos con los que hablamos de esto- pero no sé cómo aplicar $\mathrm{OOO}$ a la arquitectura o a la literatura o a las artes. (Harman, 2013, 52'00"-52'19").

\section{Agradecimientos}

Este artículo se desarrolló gracias a la colaboración de los estudiantes del semillero de investigación en teoría arquitectónica que coordina el autor en la Universidad Antonio Nariño, en particular en el proceso de documentación y revisión bibliográfica de las fuentes, las cuales se discuten siguiendo la metodología de seminario a lo largo del semestre. 


\section{Referencias bibliográficas}

- Barrera, C. (2018, 1 de julio). Pansexuales: más allá de género. Revista Credencial. Recuperado de http://www. revistacredencial.com/credencial/noticia/actualidad/ pansexuales-mas-alla-del-genero

- Bogost, I., Bryant, L., Harman, G. y Shaviro, S. (23 de Abril de 2010). Object Oriented Ontology. Atlanta, Estados Unidos: School of Literature, Communication and Culture, Georgia Institute of Technology. Recuperado de http://ooo.gatech.edu

- Bryant, L. R. (2011). The democracy of object. Ann Harbor, Estados Unidos: Open Humanities Press.

- Candia, C. (2007). Filosofía, identidad y pensamiento político en Latinoamérica. Polis. Revista Latinoamericana, (18). Recuperado de https://journals.openedition. org/polis/4054

- Carpo, M. (2001). Architecture in the age of printing: orality, writing, typography, and printed images in the history of architectural theory. Cambridge, Reino Unido: Cambridge University Press.

- Chiarella, M. (2011). Pliegues, despliegues y repliegues Didáctica proyectual e instrumentos de Ideación. Arquiteturarevista, 7(1), 63-72.

- Claro. (2017a). Internet of things. Recuperado de http://www.claro.com.co/personas/servicios/promociones/339/?gclid=EAlalQobChMIs9iXI9nm1gIVw1mG Ch370gM6EAAYASAAEgLdkfD_BwE

- Claro. (2017b). Internet of things. Recuperado de http://www.claro.com.co/negocios/soluciones/ promociones/332/

- DeLanda, M. (30 de marzo de 2002). A new ontology for the social sciences. Urbana-Champaign, Illinois, Estados Unidos: University of Illinois.

Diccionario de la Real Academia Española, DRAE (2015). Por [Definición]. Madrid, España: Asociación de academias de la lengua Española. Recuperado de http:// dle.rae.es/?id=TgJ7yhD

- Fisher, S. (9 de septiembre de 2015). Philosophy of architecture. The Stanford Encyclopedia of Philosophy. Recuperado de https://plato.stanford.edu/entries/ architecture/\#ArcRelNeg

- Gage, M. (2014). Theory through objects. Connecticut, Estados Unidos: Yale School of Architecture. Recuperado de http://architecture.yale.edu/courses/theory-throughobjects

- Galloway, A. (2013). The poverty of philosophy: realism and post-fordism. Critical Inquiry, 39(2), 347-366.
- Gandelsonas, M. (1998). The city as the object of architecture. Assemblage, 37(1), 128-144.

- Gender Agenda. (2014). Definition of Trans/TIQ. Recuperado de http://www.genderagenda.net/ definition.htm

- Gutiérrez, R. y Gutiérrez-Viñuales, R. (2012). Una mirada crítica a la arquitectura latinoamericana del siglo XX: de las realidades a los desafíos. En: E. Karge (ed.). 18101910-2010. Independencias dependientes. Art and national identities in Latin America. Dresde, Alemania: Universidad de Dresde.

- Hale, J. (29 de mayo de 2013). Harman on Heidegger: 'buildings as tool-beings'. Body of theory. Recuperado de https://bodyoftheory.com/2013/05/29/harman-on-heidegger-buildings-as-tool-beings/

- Harman, G. (2002). Tool-being: Heidegger and the metaphysics of objects. Chicago, Estados Unidos: Open Court.

- Harman, G. (23 de abril de 2007). Objects, a brief description. Londres, Reino Unido: AA School of Architecture. Recuperado de https://www.youtube.com/ watch $? v=400 q G o 3$ YHA\&t=1383s

- Harman, G. (2009). Prince of networks: Bruno Latour and metaphysics. Melbourne, Australia: Re.press.

- Harman, G. (2010a). I am also of the opinion that materialism must be destroyed. Environmental and Planning D: Society and Space, 28(1), 772-790.

- Harman, G. (2010b). Towards speculative realism: essays and lectures. Washington, D.C., Estados Unidos: Zero Books.

- Harman, G. (2012). The well-wrought broken hammer: object-oriented literary criticism. New Literary History, 43(2), 183-203.

- Harman, G. (22 de junio de 2013). What objects mean for architecture. The Swedenborg society, The Architecture Exchange, Seminar Series 1. Recuperado de http:// thearchitectureexchange.com/series-1/

- Hays, K. M. (2004). Architecture's desire: reading the late avant-garde. Cambridge and London: MIT Press.

- Heidegger, M. (1962). Being and time. New York, Estados Unidos: Harper and Row.

- Holland, O. (19 de noviembre de 2018). The architect transforming cities into 'vertical forests'. CNN Edition. Recuperado de http://edition.cnn.com/style/article/riba-vertical-forest-stefano-boeri/index.html

- Jacobs, K., y Gabriel, M. (2013). Introduction: homes, objects and things. Housing, Theory and Society, 30(3), 213-218. 
- Korody, N. (11 de marzo de 2016). Timothy Morton on haunted architecture, dark ecology and other objects. Archinect. Recuperado de https://archinect.com/features/ article/149934079/timothy-morton-on-haunted-architecture-dark-ecology-and-other-objects

- Latour, B. (22 de febrero de 2011). Do objects reside in res extensa and if not where are they located? Londres, Reino Unido: AA School of Architecture. Recuperado de https://www.youtube.com/watch?v=PFM6aFm3wDc\& $\mathrm{t}=2242 \mathrm{~s}$

- Marr, B. (4 de enero de 2018). The Internet of Things (IOT) will be massive in 2018: here are the 4 predictions from IBM. Forbes. Recuperado de https://www.forbes. $\mathrm{com} /$ sites/bernardmarr/2018/01/04/the-internet-ofthings-iot-will-be-massive-in-2018-here-are-the-4-predictions-from-ibm/\#26113f98edd3

- Matthews, G. J. (1990). Aristotelian essentialism. Philosophy and Phenomenological Research, 50(1), 251 262.

- Meillasoux, Q. (2008). After finitude. An essay on the necessity of contingency. Londres y Nueva York: Continuum.

- Microsoft. (2009). Object-Oriented Architectural Style. Microsoft Application Architecture Guide (2nd Edition) Recuperado de https://msdn.microsoft.com/en-us/library/ee658117.aspx

- Morton, T. (2010). The ecological thought. Cambridge y Londres: Harvard University Press.

- Muñoz, M. (2007). Gazapos del lenguaje arquitectónico contemporáneo colombiano. Nodo, 2(1), 41-62.

- Pallasmaa, J. (21-23 de agosto de 1992). Identity, intimacy and domicile: notes on the phenomenology of home. College of information science and technology, Pennsylvania State University. Recuperado de http://citeseerx ist.psu.edu/viewdoc/download; jsessionid=4C2E1835A9FDD96D0EAC17408C830502?doi=10.1.1.475.7009\& re rep=rep $1 \&$ type $=p d f$

- Ptolemy, B. (productor) y Ptolemy, B. (director). (2009) Transcendent man [cinta cinematográfica]. United States: Ptolemaic Productions, Therapy Studios.

- Ruy, D. (30 de enero de 2013). Returning to (strange) objects. Los Ángeles, Estados Unidos: Southern California Institute of Architecture. Recuperado de https://www. youtube.com/watch?v=bxAbiNu9Xhwe

- Schumacher, P. (2012). Architecture's next ontologica innovation. En S. Ruel-Bergeron. (Ed.), Tarp. No Nature (pp. 100-107). New York, Estados Unidos: Pratt Institute.

- Thill, S. (31 de marzo de 2011). March 31, 1999 the matrix hooks us. San Francisco, Estados Unidos: Wired Magazine. Recuperado de: https://www.wired. com/2011/03/0331the-matrix-film-released/
- Till, J. (2009). Architecture Depends, Volume 55 Cambridge: MIT Press.

- Vedral, V. (22 de marzo de 2010). Vlatko Vedral: everything is information. Londres, Reino Unido: The Guardian. Recuperado de http://www.internet-history. info/media-library/mediaitem/2435-everything-isinformation-vlatko-vedral.html

- Vitruvio, M. (1995). Los diez libros de arquitectura Madrid, España: Alianza Forma.

- Waisman, M. (1989). Un vacío hermético. Meditaciones inútiles acerca de la crisis. Arquitectura Viva, 8, 62.

- Whitehead, A. N. (1978). Process and reality. New York, Estados Unidos: Free Press.

- Xia, F., Yang, L.T., Wang, L., y Vinel, A. (2012). Internet of Things. International Journal of Communications Systems, 25(1),1101-1102.

- Yaneva, A. (2009). The making of a building: a pragmatist approach to architecture. Londres, Reino Unido: Peter Lang.

- Zizek, S. (2017). The courage of hopelessness: chronicles of a year of acting dangerously [Libro electrónico]. Londres, Reino Unido: Penguin Books Limited. Recuperado de https://books.google.com.co/books/ about/The Courage of Hopelessness.html?id=DC eDQAAQBAJ\&redir_esc $=y$

- Zizek, S. (5 de noviembre de 2018). Modalities of the absolute [Audio podcast]. Birbeck, Reino Unido: University of London. Recuperado de http:// zizekpodcast.com/2018/11/06/ziz216-modalities-of-theabsolute-05-11-2018/ 